\title{
A Report of the Efforts of the Veterans Health Administration National Antimicrobial Stewardship Initiative
}

\author{
Allison A. Kelly, MD, MSOH; ${ }^{1,2,3}$ Makoto M. Jones, MD, MS; ${ }^{4,5}$ Kelly L. Echevarria, PharmD; $;, 7,8$ \\ Stephen M. Kralovic, MD, MPH; $;{ }^{1,2,3}$ Matthew H. Samore, MD; $;{ }^{4,5}$ Matthew B. Goetz, MD; ;,10 \\ Karl J. Madaras-Kelly, PharmD, MPH; ${ }^{11,12}$ Loretta A. Simbartl, MS; ${ }^{1}$ Anthony P. Morreale, MBA, PharmD, BCPS; ${ }^{13}$ \\ Melinda M. Neuhauser, PharmD, MPH; ${ }^{14}$ Gary A. Roselle, $\mathrm{MD}^{1,2,3}$
}

овjестіve. To detail the activities of the Veterans Health Administration (VHA) Antimicrobial Stewardship Initiative and evaluate outcomes of the program.

DESIGN. Observational analysis.

SETting. The VHA is a large integrated healthcare system serving approximately 6 million individuals annually at more than 140 medical facilities.

METHODs. Utilization of nationally developed resources, proportional distribution of antibiotics, changes in stewardship practices and patient safety measures were reported. In addition, inpatient antimicrobial use was evaluated before and after implementation of national stewardship activities.

RESULTS. Nationally developed stewardship resources were well utilized, and many stewardship practices significantly increased, including development of written stewardship policies at $92 \%$ of facilities by $2015(P<.05)$. While the proportional distribution of antibiotics did not change, inpatient antibiotic use significantly decreased after VHA Antimicrobial Stewardship Initiative activities began $(P<.0001)$. A $12 \%$ decrease in antibiotic use was noted overall. The VHA has also noted significantly declining use of antimicrobials prescribed for resistant Gramnegative organisms, including carbapenems, as well as declining hospital readmission and mortality rates. Concurrently, the VHA reported decreasing rates of Clostridium difficile infection.

conclusions. The VHA National Antimicrobial Stewardship Initiative includes continuing education, disease-specific guidelines, and development of example policies in addition to other highly utilized resources. While no specific ideal level of antimicrobial utilization has been established, the VHA has shown that improving antimicrobial usage in a large healthcare system may be achieved through national guidance and resources with local implementation of antimicrobial stewardship programs.

Infect Control Hosp Epidemiol 2017;38:513-520

The Centers for Disease Control and Prevention (CDC) estimates that 2 million people annually acquire infections due to antimicrobial-resistant organisms in the United States. ${ }^{1}$ Increasing resistance and decreased development of effective therapeutics create challenges in the management of infectious diseases. Antimicrobial use is a strong risk factor for the development of antimicrobial-resistant infections and may also lead to unintended consequences such as adverse drug events and infection due to Clostridium difficile. ${ }^{2-5}$ Antimicrobial stewardship programs (ASPs) strive to optimize antimicrobial use to avoid unintended consequences and improve clinical outcomes.

The Department of Veterans Affairs (VA) Veterans Health Administration (VHA) recognized the importance of

Affiliations: 1. National Infectious Diseases Service, Veterans Health Administration, Washington, DC; 2. Cincinnati Veterans Affairs Medical Center, Cincinnati, Ohio; 3. University of Cincinnati College of Medicine, Cincinnati, Ohio; 4. IDEAS Center, Salt Lake City Veterans Affairs Medical Center, Salt Lake City, Utah; 5. University of Utah School of Medicine, Salt Lake City, Utah; 6. South Texas Veterans Healthcare System, San Antonio, Texas; 7. University Texas Health Science Center, San Antonio, Texas; 8. University of Texas, Austin, Texas; 9. Veterans Affairs Greater Los Angeles Healthcare System, Los Angeles, California; 10. David Geffen School of Medicine, University of California Los Angeles, Los Angeles, California; 11. Boise Veterans Affairs Medical Center, Boise, Idaho; 12. College of Pharmacy, Idaho State University, Meridian, Idaho; 13. Pharmacy Benefits Management Services, Veterans Health Administration, Washington, DC; 14. Pharmacy Benefits Management Services, Veterans Health Administration, Hines, Illinois.

PREVIOUS PRESENTATION: A portion of the data presented in this manuscript was presented as Abstract no. 37663 at ID Week 2012 on October 19, 2012 , in San Diego, California.

Received October 10, 2016; accepted December 14, 2016; electronically published January 25, 2017

(c) 2017 by The Society for Healthcare Epidemiology of America. All rights reserved. 0899-823X/2017/3805-0001. DOI: 10.1017/ice.2016.328 
antimicrobial stewardship programs and began the VHA Antimicrobial Stewardship Initiative in mid-2010 with the goal of providing national guidance and resources for the implementation of ASPs at local VHA medical centers (VAMCs). This initiative has been coordinated through the VHA National Antimicrobial Stewardship Task Force (ASTF) chartered in May 2011. The ASTF, cochaired by representatives from the VHA National Infectious Diseases Service and the National Pharmacy Benefits Management Services, is comprised of a 25 -member multidisciplinary team who were selected based on their areas of expertise. In January 2014, with the publication of VHA Directive 1031, which requires all VHA medical facilities to implement, maintain, and annually evaluate ASPs, the VA solidified its commitment to optimize antimicrobial usage and improve the care of veterans. ${ }^{6}$

The purpose of this study was to detail the activities of the VHA Antimicrobial Stewardship Initiative and to evaluate the national process and outcome measures of the program.

\section{METHODS}

\section{Setting}

The VHA is a large, federal, integrated healthcare system serving approximately 6 million individual patients annually at more than 140 medical facilities distributed throughout the country. VHA medical facilities offer a wide range of acute care, long-term care, outpatient services, and rehabilitation services to enrolled veterans across the United States. Veteran population demographics and VHA medical facility characteristics are provided in Supplemental Tables 1 and 2.

\section{Engagement: Education and Information Gathering}

The VHA Antimicrobial Stewardship Initiative began with a series of educational conferences presented by national subject matter experts to discuss the concept of stewardship with interested VHA field practitioners. This series consisted of a face-to-face conference repeated in 3 separate geographic regions to ensure systemwide representation from May through July of 2010 and a follow-up conference in November 2011, which focused more specifically on the implementation of stewardship activities and the development of ASP networks.

A number of additional stewardship activities were undertaken in 2011. A nationwide group of approximately 250 volunteer field stewardship champions, representing diverse disciplines including clinical pharmacists and infectious diseases physicians, was assembled to serve as a resource for communication and dissemination of stewardship topics and to provide multiple stewardship "best practices" documents. To aid in antimicrobial optimization, the online version of The Sanford Guide To Antimicrobial Therapy, an authoritative reference on antimicrobial therapy, was provided for use at all VHA medical facilities beginning in October 2011. Also in October 2011, the ASTF administered a voluntary self-reported survey of stewardship practices across the VA to gain an understanding of existing ASPs and to determine which ASTF activities and resources might be most useful.

In January 2012, the ASTF implemented 2 additional key activities. An internal VHA Antimicrobial Stewardship Microsoft SharePoint site was launched to serve as a robust repository of stewardship documents, resources, and tools used in the development and expansion of local stewardship programs. The ASTF also launched an ongoing monthly series of educational web-based teleconferences (eg, webinars) that covered a range of infectious diseases clinical topics and implementation strategies pertinent to stewardship.

To follow up on the 2011 voluntary survey of stewardship practices, a required comprehensive systematic survey, performed with the assistance of the VHA Healthcare Analysis and Informatics Group, was administered to each VHA facility in November 2012. This survey was revised and performed again in December 2015 to evaluate uptake and progress of stewardship activities in the field.

\section{Encouragement: Policy Development and Dissemination}

Beginning in 2012, a series of stewardship-related example policies and intervention tools, itemized in Table 1, were developed by the ASTF. The general approach to drafting these documents involved conducting a literature search of existing evidence and, when possible, performing a retrospective review of VHA practice patterns in areas targeted for intervention. Many of these policies were created within a framework that utilized antimicrobial stewardship champions, particularly clinical pharmacists, to identify appropriate patients and to make recommendations to optimize therapy. These example policies and intervention tools were subsequently "launched" through the monthly webinar series and were posted to the VHA Antimicrobial Stewardship Microsoft SharePoint site for elective adaptation and implementation as facilities deemed appropriate.

The ASTF coordinated with VA Central Office to encourage local facility institutional support for stewardship. Leadership support for ASPs was demonstrated at the highest levels with the VA Under Secretary for Health's Information Letter on Antimicrobial Stewardship published in July $2012^{7}$. This letter, distributed to all VAMCs, described the importance of ASPs and encouraged their development in the VA. Additionally, a memorandum was distributed through the VA operational hierarchy in September 2013 encouraging local administration at each VAMC to provide funding for clinical pharmacists to complete antimicrobial stewardship certificate programs based on need. Clinical pharmacists were to be selected locally for certificate programs based on training, background, and job requirements. VA Central Office leadership confirmed their commitment to stewardship with the publication of VHA Directive 1031: Antimicrobial Stewardship Programs in January $2014 .^{6}$ This directive required all VAMCs to identify local stewardship champions and to implement or augment a written 
ASP policy by July 2014 and to evaluate performance of the ASP no later than January 2015. From inception, the VHA Antimicrobial Stewardship Initiative's national subject matter expert consultants, in addition to the previously stated resources, assisted in the development of local ASPs.

\section{Data Sources}

Resource utilization data. Attendance at face-to-face educational meetings and "attendee lines" used for webinars were tallied at the time of the events. Attendee lines represent the minimal number of attendees because multiple individuals may have viewed a presentation together using a single line. Utilization of computerized stewardship resources was obtained through each individual website's total log-in data per month. Individual VAMC stewardship activities were identified through the 3 distinct national surveys conducted in 2011, 2012, and 2015.

Antimicrobial use data. The VA's Office of Information and Technology built the corporate data warehouse (CDW) to standardize and consolidate VA clinical data including bar code medication administration data (BCMA) for administered medications incorporated in the VA electronic medical record. The CDW was used to obtain BCMA data for the calculation of inpatient antimicrobial days of therapy (DOT). A DOT was defined as a single dose of an antimicrobial given intravenously or orally to a patient on a single day regardless of the strength or frequency of the drug. ${ }^{8}$ For example, administration of vancomycin as a single 1-g dose or as two 1-g doses given 12 hours apart are both considered 1 DOT. DOT for an antibiotic with possible intravenous and oral forms combined both formulations in the usage data for that agent. Nationwide VAMC inpatient bed days of care (BDOC) were obtained from the VA's Austin Information Technology Center data center. A BDOC was defined as a day during which a person was confined to a bed where the patient stayed overnight. Inpatient status was defined as an admission to an acute-care bed, including an intensive care unit, step-down unit, medical unit, or surgical unit, in addition to observational bed status. The antimicrobial use calculations excluded hemodialysis, psychiatric, rehabilitation, or longterm care nursing units. Days of therapy per 1,000 BDOC by quarter were evaluated for all antibiotics from 2007 through 2015. All antibiotic use data were analyzed in an aggregated deidentified manner. Notably, at no time during the analysis period did the VHA experience any significant national formulary changes or prolonged shortages of antibiotics.

For dissemination of these data beyond programmatic needs, the Institutional Review Board of the Cincinnati VA Medical Center evaluated our program analysis.

\section{Statistics}

Using SAS version 9.3 (SAS Institute), linear regression analysis was used to examine the progression of the DOT per
1,000 BDOC by quarter for the time periods before and after inception of the VHA Antimicrobial Stewardship Initiative. A comparison of the linear regression slopes was performed to determine any significance in the difference of the slopes for the 2 periods. We used $\chi^{2}$ analyses to examine the proportional distribution of antibiotic use for specific antibiotics and antibiotic classes or groups from 2007 to 2015. In addition, $\chi^{2}$ analyses were used to determine the differences in selected stewardship activities and reported challenges from the 2011, 2012, and 2015 surveys.

\section{RESULTS}

Process Measures: Utilization of Resources and Stewardship Practices Nationwide

Together, the VHA Antimicrobial Stewardship Initiative's face-to-face educational conferences hosted nearly 450 participants from VHA facilities evenly distributed throughout the country. The online version of The Sanford Guide and the VHA Antimicrobial Stewardship Microsoft SharePoint site experienced consistently increasing utilization, with an average number of monthly visits of $>5,000$ and 2,400, respectively. In addition, the ASTF monthly webinars were well attended, with an average of $>175$ attendee lines used per month (Figure 1). As shown in Table 1, the ASTF example policies were utilized at varying rates, with a high of $51 \%$ of facilities with intravenous to oral conversion interventions reporting use of that ASTF example policy in the development of local processes.

The initial 2011 ASTF Inventory of Stewardship Practices indicated that each of the 126 respondent acute-care VAMCs performed at least 1 stewardship activity, and $>70 \%$ of facilities performed the following activities: generation of annual antibiograms, imposed formulary restrictions, policies for criteria for use of certain antimicrobials, utilization of inpatient clinical pathways or guidelines, selective microbiology result reporting and dose optimization of selected antimicrobials. The subsequent 2012 and 2015 surveys, completed by 130 and 140 VAMCs respectively, demonstrated progressive increases in numerous additional stewardship practices and decreases in challenges reported by ASPs. As of the 2015 Stewardship Survey, $89 \%$ of facilities had defined a stewardship team; this number had increased from $41 \%$ in 2011 $(P<.05)$. Furthermore, $92 \%$ of facilities had a written policy for stewardship, which had increased from $17 \%$ in 2011 $(P<.05)$. The results of selected stewardship practices and challenges with improvements from the 2011, 2012 and 2015 surveys are summarized in Figures 2 and 3.

\section{Outcome Measures: Nationwide VHA Antimicrobial Use, Resistance, and Patient Safety}

Aggregated quarterly inpatient antibiotic use (Figure 4), significantly decreased after VHA Antimicrobial Stewardship Initiative activities began in the second quarter of 2010 $(P<.0001)$. A $12 \%$ decrease in antibiotic use was noted 


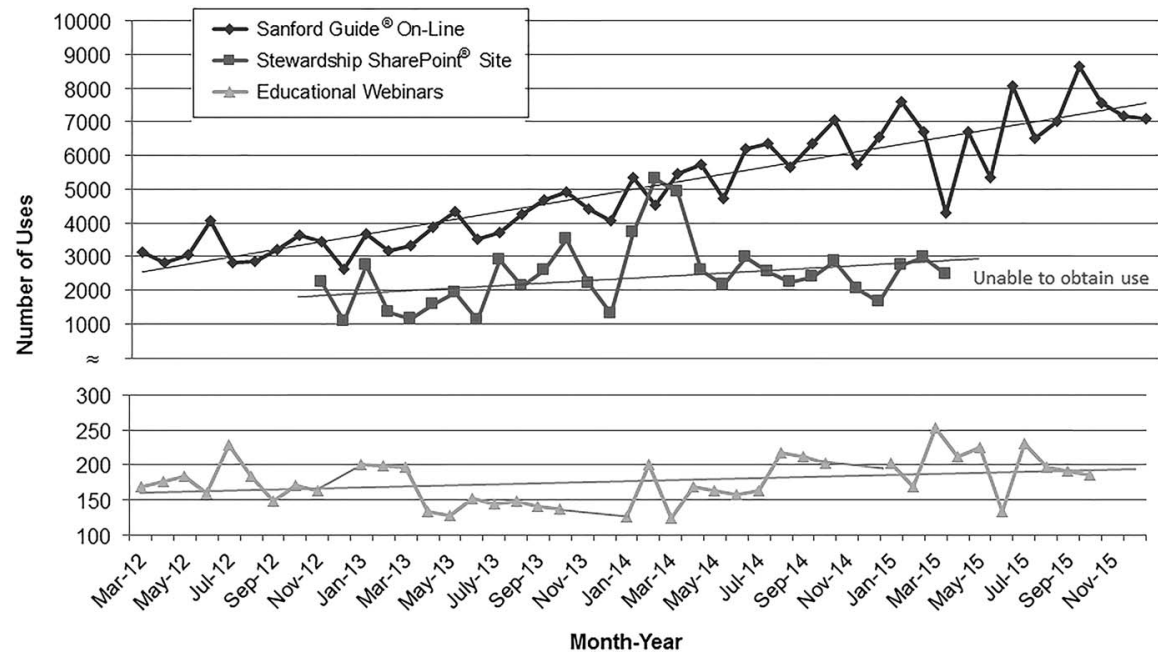

FIGURE 1. Monthly use of nationally provided resources. Total monthly log in usage data for electronic resources provided by the VHA Antimicrobial Stewardship Initiative and attendee lines used for stewardship educational webinars. Note that usage data for the VHA Antimicrobial Stewardship SharePoint site became unavailable after March 2015 due to a website platform change.

тав LE 1. Antimicrobial Stewardship Task Force (ATSF) Example Policies and Interventions

\begin{tabular}{|c|c|c|c|}
\hline Example Policies & Launch Date & $\begin{array}{l}\text { Facilities, No. }(\%) \\
\qquad(\mathrm{n}=140)\end{array}$ & $\begin{array}{c}\text { that Utilized ASTH } \\
\text { Examples, } \%{ }^{\mathrm{b}}\end{array}$ \\
\hline Intravenous to oral conversion tool & May 2012 & $116(83)$ & 51 \\
\hline Avoidance of double anaerobic coverage & Jun 2012 & $99(71)$ & 42 \\
\hline Stewardship monitoring of outpatient parenteral antimicrobial therapy & Nov 2012 & $85(61)$ & 22 \\
\hline Vancomycin de-escalation & Jan 2013 & $97(69)$ & 32 \\
\hline Workload documentation guidance & May 2013 & $64(46)$ & 27 \\
\hline Pneumonia duration of therapy & Sep 2014 & $63(45)$ & 21 \\
\hline S. aureus bacteremia intervention & Jul 2015 & $83(59)$ & 20 \\
\hline
\end{tabular}

${ }^{\mathrm{a}}$ Facilities that reported performing the specified interventions on the 2015 Stewardship Survey.

${ }^{\mathrm{b}}$ Percentage of facilities performing the specified intervention that reported utilizing the ASTF example policies to develop the intervention.

overall, from a quarterly high of 761.2 DOT per 1,000 BDOC in the first quarter of 2008 , prior to the Initiative, to 673.3 DOT per 1,000 BDOC in the final quarter of 2015.

Use of broad-spectrum antimicrobials for highly resistant organisms (eg, carbapenems, polymyxins, and tigecycline) are potential surrogates for resistance given their role in the treatment of such resistant organisms. All 3 of these antimicrobial classes/agents demonstrated significantly increased use prior to the VHA Antimicrobial Stewardship Initiative, and a decreasing trend in the use of carbapenems and significantly decreased use of polymyxins and tigecycline was noted after the Initiative began. (Figure 5) A comparison of the linear regression slopes revealed significant decreases in use of each of the antimicrobial classes/agents $(P<.05$ for each).

Vancomycin, fluoroquinolones, piperacillin-tazobactam, and cephalosporins were consistently the most frequently administered antibiotics or antibiotic classes from January 2007 through December 2015, with vancomycin and piperacillintazobactam alone consistently totaling nearly one-third of all antibiotic use. The proportion of fluoroquinolone use dropped from $18 \%$ in 2007 to $13 \%$ in 2015 , and the proportion of cephalosporin use increased from $16 \%$ in 2007 to $22 \%$ in 2015, but neither trend was significant ( $P=.95$ and .97 , respectively). Percentage distributions of the remainder of the antibiotic agents, classes, and groupings remained stable throughout the period, with no significant differences observed across the years $(P=.99)$ (Supplemental Figure 1).

The VHA Multidrug-Resistant Organism Prevention Initiative, a highly active complementary national program, maintains a specific prevention initiative for Clostridium difficile infection, which incorporates a bundled approach of infection control and environmental management practices 


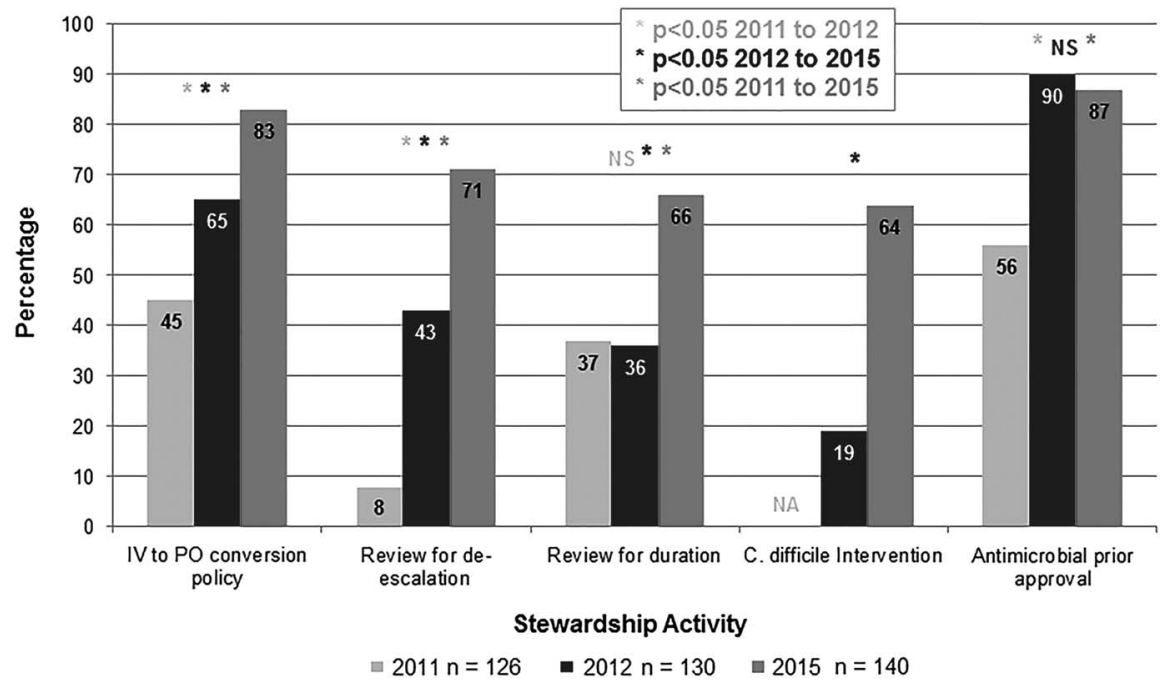

FIGURE 2. Comparison of selected stewardship practices. Percentage of reported activities at VHA medical centers from surveys completed in 2011, 2012, and 2015 compared using $\chi^{2}$ analyses. Abbreviations: IV, intravenous; PO, oral; NS, nonsignificant.

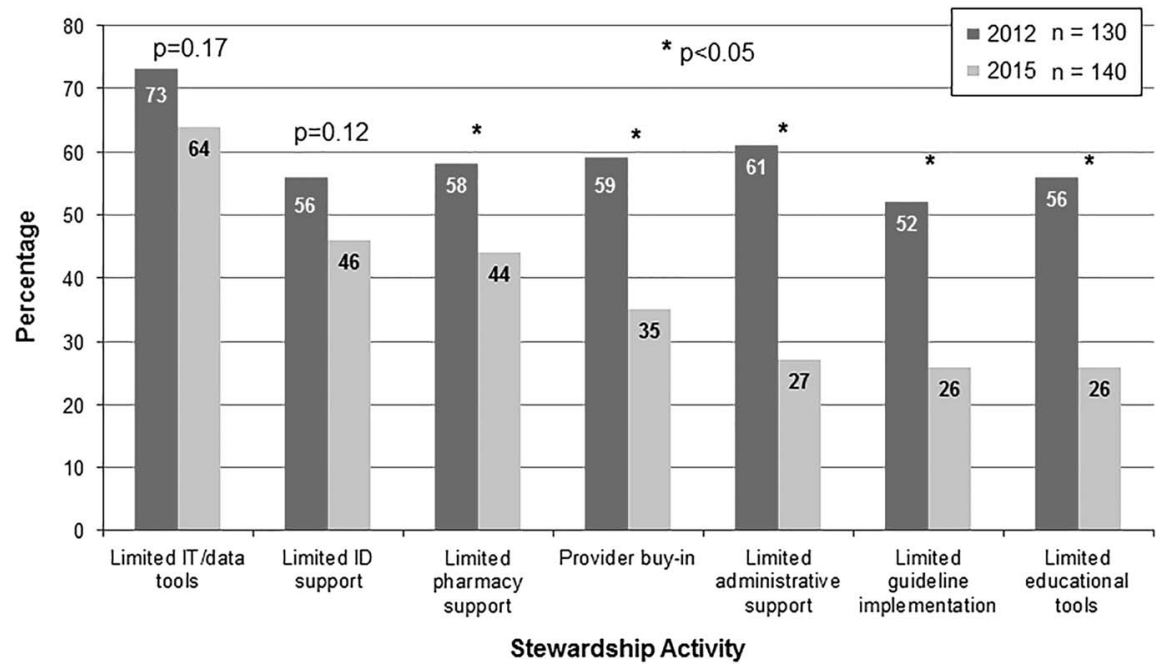

FIGURE 3. Comparison of reported challenges to stewardship programs. Percentage of reported challenges to stewardship programs incurred at VHA medical centers from surveys completed in 2012 and 2015 compared using $\chi^{2}$ analyses. Abbreviations: IT, information technology; ID, infectious diseases.

with cultural transformation, and was implemented July 2012. This parallel program has published decreasing rates of C. difficile infection in both acute-care and long-term-care VHA facilities in the same time period as concomitant activity by the VHA Antimicrobial Stewardship Initiative. ${ }^{9,10}$

Hospital acute-care and intensive-care lengths of stay remained stable, while readmission and mortality rates decreased after the implementation of the VHA Antimicrobial Stewardship Initiative (Supplemental Table 3). Total hospital discharges related to infectious diseases as determined by diagnosis related group increased from 2012 to 2014, and the percentage distribution of those discharges by infectious diseases diagnosis group remained stable (Supplemental Figure 3).

\section{I SCUSSION}

Antimicrobial use is a known driver of antimicrobial resistance with recent reports showing that global antibiotic consumption increased by $36 \%$ between 2000 and $2011 .{ }^{11}$ As much as half of all antibiotic use is potentially unnecessary, so antimicrobial stewardship programs with methods to optimize antimicrobial use have become essential. ${ }^{12-14}$ To this end, the VHA has instituted a successful National Antimicrobial Stewardship Initiative coordinated through its National Antimicrobial Stewardship Task Force. The Task Force has developed effective, highly utilized educational programs, tools, and resources to support the implementation and 


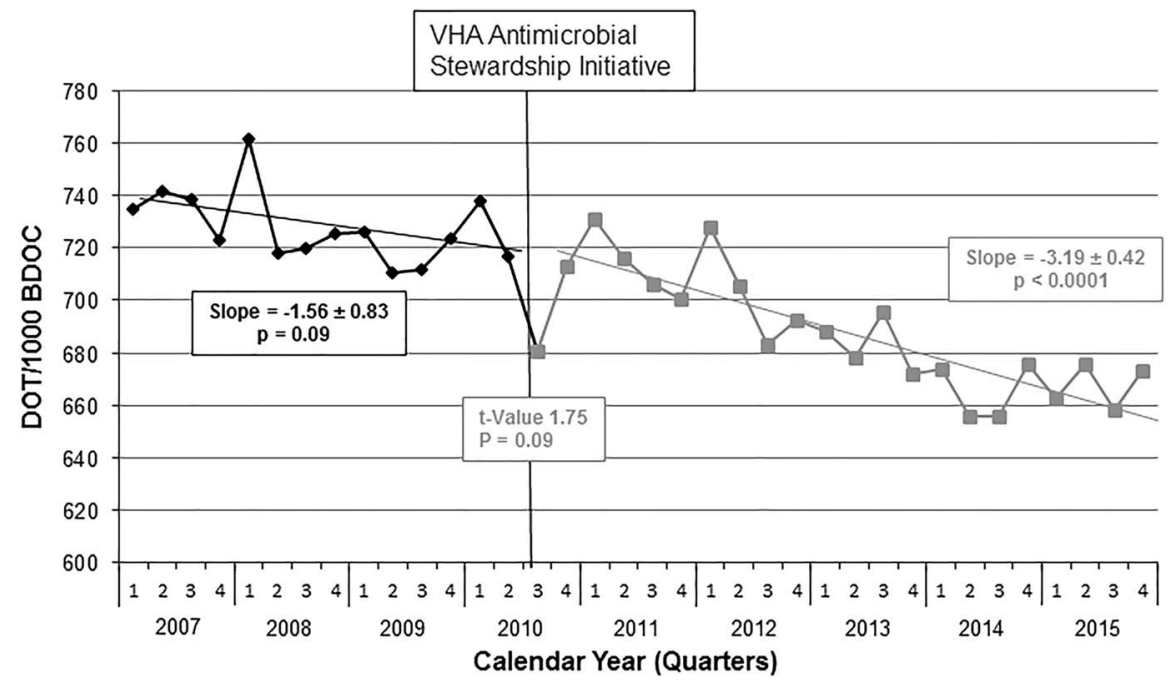

FIGURE 4. Veterans Health Administration (VHA) inpatient antibiotic use: days of therapy (DOT) per 1,000 bed days of care (BDOC). Inpatient antibiotic use before and after activities for the VHA Antimicrobial Stewardship Initiative began in the second quarter of calendar year 2010 (denoted by the vertical black line). Analysis was performed using simple linear regression with comparison of slopes. An overall $12 \%$ decline in use was observed. Abbreviations: DOT, days of therapy; BDOC, bed days of care.

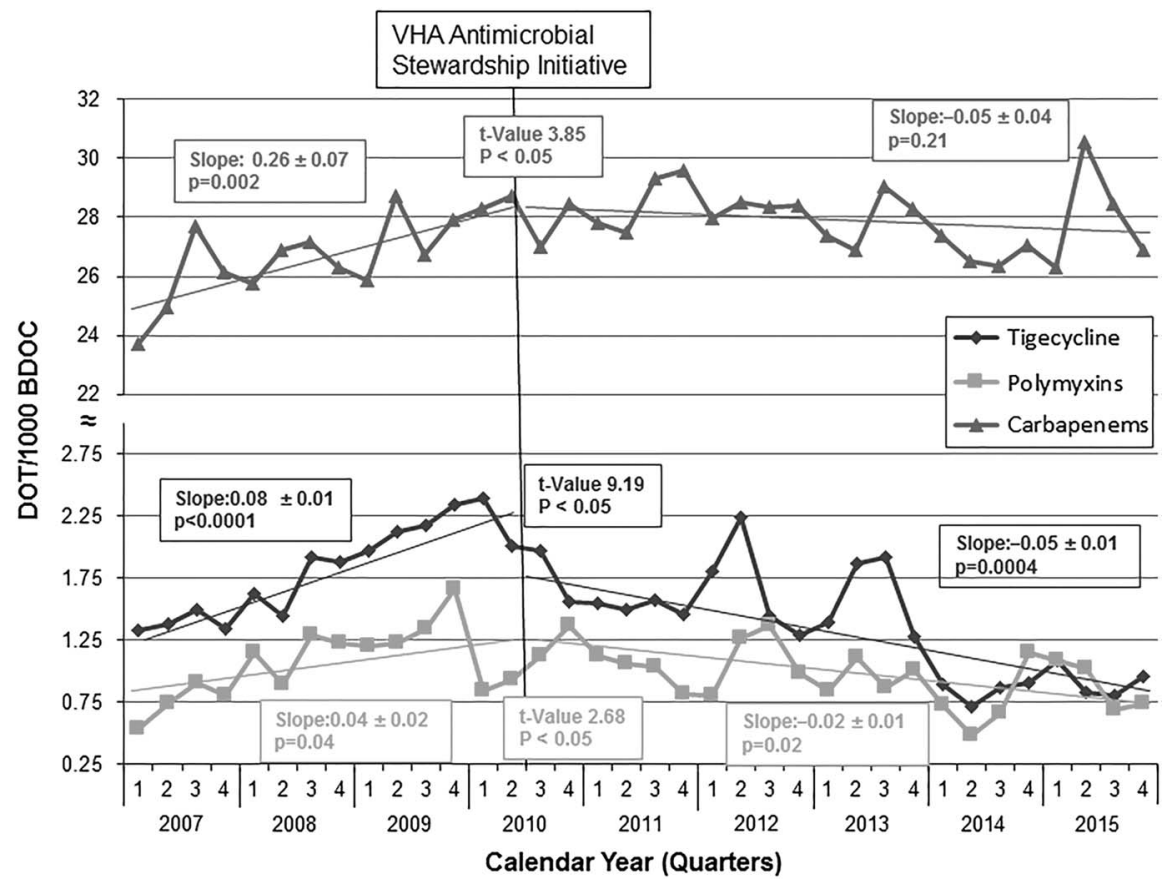

FIGURE 5. Inpatient use of selected antibiotics targeting resistant organisms. Carbapenem, polymyxin, and tigecycline use before and after implementation of the VHA Antimicrobial Stewardship Initiative in the second quarter of calendar year 2010 (denoted by the vertical black line). Analysis was performed using simple linear regression with comparison of slopes. Abbreviations: DOT, days of therapy; BDOC, bed days of care.

augmentation of local VHA ASPs. This task force has also spearheaded publication of VHA national policies regarding stewardship. VHA national stewardship policies allow VAMCs to utilize local experts and resources to optimize and individualize their ASPs. After the VHA National Antimicrobial
Stewardship Initiative began, inpatient antimicrobial use declined significantly by $12 \%$ overall, with 673.3 DOT per 1,000 BDOC noted in the final quarter of 2015. No change was observed in the yearly proportional distribution of specific antimicrobial agents, classes, and groups. This finding 
intimates that the decrease in antimicrobial use was not related specifically to 1 agent or 1 class but was more an overall global decline in antimicrobial use across antimicrobial classes. Additionally, from 2012 to 2015, the VA has noted decreasing rates of $C$. difficile infection and significantly declining use of antimicrobial agents prescribed for highly resistant Gramnegative organisms, including carbapenems. ${ }^{9,10}$ During this same period, improvements in VHA medical facility readmission and mortality rates were observed.

Few reports in the literature have described antimicrobial use in a large diverse healthcare system such as the VA health-care system. Polk et al, ${ }^{15}$ working with the University HealthSystem Consortium, reported on antibiotic use in 2009 from 70 academic medical centers in the United States. ${ }^{15}$ These researchers found a mean hospital-wide use of 839.0 DOT per 1,000 patient days, whereas inpatient VHA antimicrobial usage for that calendar year was considerably lower at 718.1 DOT per 1,000 BDOC. More recently, Baggs et $\mathrm{al}^{16}$ published an analysis of inpatient antibiotic use from 2006 to 2012 demonstrating an overall national DOT of 755 per 1,000 patient days, which is higher than VHA's average quarterly use from 2007 to 2012 of 718.6 DOT per 1,000 BDOC. In addition, these researchers estimated significant increases in several antibiotic classes, including carbapenems, which were used for 32.3 DOT per 1,000 patient days in 2012. Carbapenem use in VHA initially increased prior to the inception of the VHA Antimicrobial Stewardship Initiative but subsequently decreased, with an average quarterly use of 28.3 DOT per 1,000 BDOC in 2012.

Our report has some limitations. At the time of our analysis, validated national microbiologic data extraction tools for VHA were not available; therefore, use of broad-spectrum antimicrobials for highly resistant organisms was employed as a surrogate for resistance. Additionally, the concomitant successful VHA MDRO C. difficile Prevention Initiative limits interpretation of the impact of the VHA Antimicrobial Stewardship Initiative on $C$. difficile rates. The VHA Antimicrobial Stewardship Initiative provides national support and resources for local implementation of stewardship interventions but does not require the implementation of specific stewardship policies and does not define appropriateness of use. All VHA facilities are encouraged to design ASPs around their unique needs and available resources. This approach and the diverse nature of VAMCs with varying levels of facility capabilities and patient complexities do not allow for analysis of the impact of specific stewardship interventions from a national perspective. In addition, we were unable to determine which interventions may be most effective at optimizing antimicrobial use to appropriate levels. VA researchers, however, are utilizing the VHA Stewardship surveys to overlay stewardship activities with antimicrobial use to define key characteristics of effective ASPs. Using the data collected from the 2012 VHA Stewardship Survey, Chou et $\mathrm{al}^{17}$ concluded that decreased antimicrobial use was associated with having infectious diseases physicians and pharmacists present, as well as the frequency of patient-level reviews of antimicrobial use, and with having a policy to address antimicrobial use in patients with $C$. difficile infection. A similar analysis of the data reported on the 2015 VHA Stewardship Survey is in process.

Over the course of several years with dedicated national and local volunteers, the VA has established stewardship programs nationwide. Through continued education, disease-specific guideline reviews, sample policy development and other resources, along with leadership engagement and support, the VHA Antimicrobial Stewardship Initiative has implemented ongoing efforts to optimize antimicrobial use to attain the goal of a $20 \%$ reduction in inpatient antibiotic use by 2020 set forth in the National Action Plan for Combatting AntibioticResistant Bacteria. ${ }^{18}$ While no specific ideal level of antimicrobial utilization is known, the VA has shown that decreasing antimicrobial usage in a large healthcare system may be achieved through national guidance and resources with local implementation of ASPs. The VHA Antimicrobial Stewardship Initiative will continue to lead in the development of stewardship programs with expansion of stewardship activities to other areas of patient care, particularly long-term and outpatient care. Hopefully, this Initiative will serve as a model for other large healthcare systems.

\section{ACKNOWLEDGMENTS}

We thank Kevin Nechodom of the IDEAS Center Salt Lake City VA Medical Center for his expert technical support. We also thank the Antimicrobial Stewardship Task Force and work group members and, most importantly, the Antimicrobial Stewardship Champions at each VHA facility across the country for their efforts in optimizing antimicrobial use and improving the health care of US Veterans.

Financial support: The authors acknowledge funding support from the Center for Disease Control and Prevention's Safety and Healthcare Epidemiology Prevention Research Development (SHEPHerD; task order 2013-03) to develop the collection of bar code medication administration data to assess antimicrobial days of therapy.

Potential conflicts of interest: All authors report no conflicts of interest related to this article.

Address correspondence to Allison A. Kelly, 205 W 4th St, Suite 1020, Cincinnati, OH 45202 (Allison.Kelly@va.gov).

SUPPLEMENTARY MATERIAL

To view supplementary material for this article, please visit https://doi.org/10.1017/ice.2016.328

\section{REFERENCES}

1. Antibiotic resistance threats in the United States. 2013. Centers for Disease Control and Prevention website. http://www.cdc.gov/ drugresistance/threat-report-2013/. Published 2013. Accessed March 20, 2015.

2. Patel G, Huprikar S, Factor SH, Jenkins SG, Calfee DP. Outcomes of carbapenem-resistant Klebsiella pneumoniae infection and the impact of antimicrobial and adjunctive therapies. Infect Control Hosp Epidemiol 2008;29:1099-1106.

3. Talon D, Bailly P, Bertrand X, Thouverez M, Mulin B. Clinical and molecular epidemiology of chromosome-mediated resistance to 
third generation cephalosporins in Enterobacter isolates in eastern France. Clin Micro Infect 2000;6:374-382.

4. Shehab N, Patel PR, Srinivasan A, Budnitz DS. Emergency department visits for antibiotic-associated adverse events. Clin Infect Dis 2008;15:735-743.

5. Chang HT, Krezolek D, Johnson S, Parada JP, Evans CT, Gerding DN. Onset of symptoms and time to diagnosis of Clostridium difficile-associated disease following discharge from an acute care hospital. Infect Control Hosp Epidemiol 2007;28:926-931.

6. VHA Directive 1031: Antimicrobial Stewardship Programs. http://www.va.gov/vhapublications/ViewPublication.asp?pub_ID= 2964. Published 2014. Accessed March 17, 2015.

7. VHA Under-Secretary for Health's (USH's) information letter on antimicrobial stewardship. http://vaww.vhaco.va.gov/pubarchives/ docs/IL-10-2012-011.pdf. Internal VA intranet website. Accessed March 17, 2015.

8. Polk RE, Fox C, Mahoney A, Letcavage J, MacDougall C. Measurement of adult antibacterial drug use in 130 US hospitals: comparison of defined daily dose and days of therapy. Clin Infect Dis 2007;44:664-670.

9. Evans ME, Kralovic SM, Simbartl LA, Jain R, Roselle GA. Effect of a Clostridium difficile infection prevention initiative in Veterans Affairs acute-care facilities. Infect Control Hosp Epidemiol 2016;37:720-722.

10. Reeves JS, Evans ME, Simbartl LA, Kralovic SM, Kelly AA, Jain R, Roselle GA. Clostridium difficile infections in Veterans Health Administration long-term care facilities. Infect Control Hosp Epidemiol 2016;37:295-300.

11. Van Boeckel TP, Gandra S, Ashok A, Caudron Q, Grenfell BT, Levin SA, Laxminarayan R. Global antibiotic consumption 2000 to 2010: an analysis of national pharmaceutical sales data. Lancet Infect Dis 2014;14:742-750.

12. Dellit TH, Owens RC, McGowan JE, et al. Infectious Diseases Society of America and the Society for Healthcare Epidemiology of America guidelines for developing an institutional program to enhance antimicrobial stewardship. Clin Infect Dis 2007; 44:159-177.

13. Hecker MT, Aron DC, Patel NP, Lehmann MK, Donskey CJ. Unnecessary use of antimicrobials in hospitalized patients: current patterns of misuse with an emphasis on the antianaerobic spectrum of activity. Arch Intern Med 2003;163: 972-978.

14. Hicks LA, Taylor TH, Hunkler RJ. US outpatient antibiotic prescribing, 2010. N Engl J Med 2013;368:1461-1462.

15. Polk RE, Hohmann SF, Medvedev S, Ibrahim O. Benchmarking risk-adjusted adult antibacterial drug use in 70 US academic medical center hospitals. Clin Infect Dis 2011;53:1100-1110.

16. Baggs J, Fridkin SK, Pollack LA, Srinivasan A, Jernigan JA. Estimating national trends in inpatient antibiotic use among US hospitals from 2006 to 2012. JAMA Intern Med 2016;176: 1639-1648.

17. Chou AF, Graber CJ, Jones M, et al. Characteristics of antimicrobial stewardship programs at Veterans Affairs hospitals: results of a nationwide survey. Infect Control Hosp Epidemiol 2016;37:647-654.

18. The White House's national action plan for combatting antibioticresistant bacteria. The White House website. https://www. whitehouse.gov/sites/default/files/docs/national_action_plan_for_ combating_antibotic-resistant_bacteria.pdf. Published 2015. Accessed November 28, 2016. 